\title{
Original Research \\ Bio-3D printing of scaffold-free osteogenic and chondrogenic constructs using rat adipose-derived stromal cells
}

\author{
Ryota Fujimoto $^{1,2}$, Daiki Murata ${ }^{1, *}$, Koichi Nakayama ${ }^{1}$ \\ ${ }^{1}$ Center for Regenerative Medicine Research, Faculty of Medicine, Saga University, 840-8502 Saga, Japan \\ ${ }^{2}$ Department of Oral and Maxillofacial Surgery, Faculty of Medicine, Saga University, 840-8501 Saga, Japan \\ *Correspondence: daiki_net_official@yahoo.co.jp (Daiki Murata) \\ Academic Editor: Piergiorgio Gentile \\ Submitted: 20 November 2021 Revised: 15 January 2022 Accepted: 18 January 2022 Published: 11 February 2022
}

\begin{abstract}
Background: Although autogenous bone implantation is considered to be the gold standard for the reconstruction of bone defects, this approach remains challenging when treating extensive bone defects (EBDs). Therefore, artificial materials (AMs) such as artificial bone and scaffolds are often used for treating EBDs. Nevertheless, complications such as material failure, foreign body reaction, and infection are common. To overcome these issues, we aimed to develop a new treatment for an EBD using scaffold-free adipose-derived stromal cells (ADSCs) to fabricate chondrogenic/osteogenic-induced constructs without AMs. Methods: ADSCs were obtained from the subcutaneous adipose tissue of 8-week-old female Wistar rats $(n=3)$ and assessed to determine their potential for multilineage differentiation into adipocytes (Oil Red O staining), chondrocytes (hematoxylin and eosin, Alcian blue, and Safranin O staining), and osteoblasts (Alizarin red and von Kossa staining). Spheroids $(n=320)$, each containing $3.0 \times 10^{4}$ ADSCs, were then used to fabricate scaffold-free cell constructs using a bio-3D printer with a needle array. The spheroids and constructs were stimulated with induction medium to induce chondrogenic and osteogenic differentiation. The induced cartilage- and bone-like constructs were finally evaluated using micro-computed tomography $(\mu \mathrm{CT})$ and histological analysis. Results: The collected ADSCs were capable of trilineage differentiation, and were successfully used to produce scaffold-free constructs. The fabricated constructs $(\mathrm{n}=3)$ exhibited equivalent strength (load, $195.3 \pm 6.1 \mathrm{mN}$; strength, $39.1 \pm 1.2 \mathrm{kPa}$; and stiffness, $0.09 \pm 0.01 \mathrm{~N} / \mathrm{mm}$ ) to that of soft tissues such as the muscles in the uninduced condition. In chondrogenic induction experiments, Alcian blue and Safranin O staining confirmed the differentiation of the constructs into cartilage, and cartilage tissue-like structures were produced. In the osteogenic induction experiment, Alizarin Red and von Kossa staining showed calcium salt deposition, and $\mu \mathrm{CT}$ images confirmed the same calcification level as that of the cortical bone. Conclusions: Scaffold-free constructs consisting of ADSCs without an AM were fabricated, and cartilage- and bone-like tissues were successfully generated, demonstrating their potential for bone reconstruction.
\end{abstract}

Keywords: osteogenic construct; scaffold-free; bio-3D printer; adipose-derived stromal cells; bone reconstruction

\section{Introduction}

As part of the skeletal system, the bone plays an important role in supporting the body. Extensive defects in the long bones are common orthopedic disorders following comminuted fractures $[1,2]$. Such extensive bone defects (EBDs) are also often observed after surgical resection of tumors of the maxillofacial bone, as it is necessary to secure a surgical margin to completely remove the tumor [3]. Autogenous bone (AB) implantation is considered to be the gold standard for the treatment of EBDs [4]. However, this method is clinically challenging owing to the limitations of bone fixation methods and its success is dependent on the size of the bone graft [5]. Therefore, in recent years, artificial materials (AMs) such as artificial bones and scaffolds have been applied to the treatment of EBDs [6-10]. Nevertheless, AMs are sometimes inferior to AB in terms of strength and integration with the surrounding bone tissue [11]. Additionally, AMs often lead to problems such as a long-term foreign body reaction after implantation and biofilm formation following bacterial infection [12]. As
AMs can also obstruct cell-cell adhesion and inhibit selforganization, it can take a long time to form mature and healthy bones [13]. A potentially effective and efficient approach to solve these problems could be to produce threedimensional (3D) bone and cartilage-like tissues using only cells without any AMs.

Many types of stem cells have been studied in parallel with development of the regenerative medicine field [14, 15]. Mesenchymal stem cells, isolated from various tissues such as the bone marrow and adipose tissue, exhibit selfproliferation and multipotency properties [16,17]. Among them, adipose-derived stromal cells (ADSCs) and stromal vascular fraction cells (SVFs) can differentiate into various cell types such as adipocytes, myoblasts, osteoblasts, chondrocytes, and neurons [18-27]. Indeed, ADSCs and SVFs have already been used for regenerative medicine [28,29]. In addition, by combining these cells with various biomaterials such as hyaluronic acid, platelet-rich plasma (PRP), and fat grafts, the range of application has been further expanded, and these cell types are thus expected to have fur- 


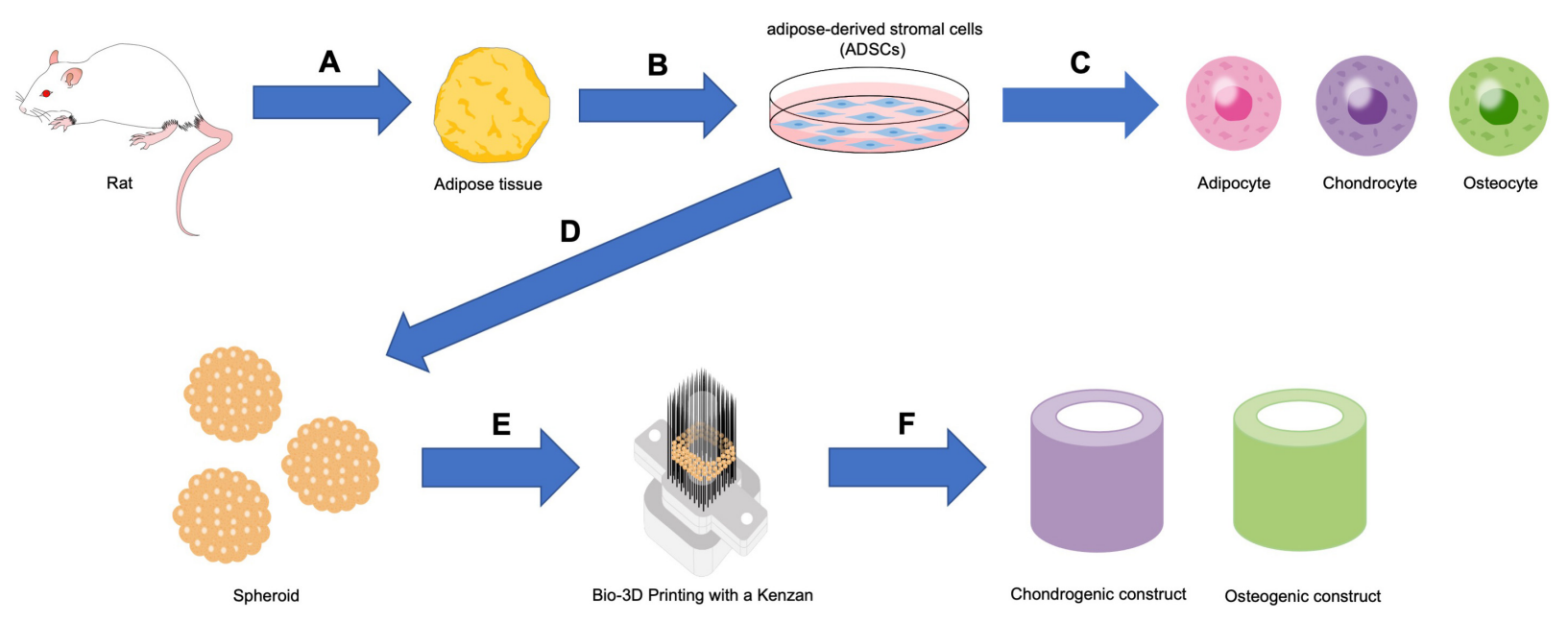

Fig. 1. Outline of this study. (A) Adipose tissue collection from rats. (B) Enzymatic treatment of adipose tissue. (C) Trilineage differentiation of adipose-derived stromal cells (ADSCs). (D) Spheroid formation of ADSCs. (E) Fabrication of a scaffold-free cellular construct using a 3D bioprinter. (F) Chondrogenic and osteogenic induction of the constructs.

ther application prospects in the future [29-34].

With the aim of developing a new bone reconstruction method for the treatment of EBDs, in this study, we used a bio-3D printer with a needle array to fabricate scaffoldfree 3D bone-like and cartilage-like constructs using only ADSCs without AMs (Fig. 1).

\section{Materials and methods}

\subsection{Animals}

Female Wistar rats ( 8 weeks old, $\mathrm{n}=3$; Charles River Laboratories Japan, Inc., Shiga, Japan) with a mean weight

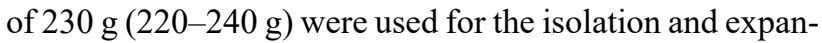
sion of ADSCs (Fig. 1A). All animals were housed under a 12-h dark-light cycle (light from 08:00 to 20:00) at 23 $\pm 2{ }^{\circ} \mathrm{C}$ and $50 \pm 10 \%$ humidity with ad libitum access to food and water. The rats were kept at a maximum of three per cage. All procedures were evaluated and approved by the Institutional Animal Care and Use Committee of Saga University (application no. A2020-048-0).

\subsection{Isolation and expansion of rat ADSCs}

Intrascapular adipose tissue $(0.5-0.8 \mathrm{~g}$ per animal) was aseptically collected under general anesthesia (induction anesthesia: 5\% isoflurane, maintenance anesthesia: 2.5\% isoflurane; Fujifilm Wako Pure Chemical Industries, Ltd., Osaka, Japan), minced, and digested for $3 \mathrm{~h}$ in phosphate-buffered saline (PBS) containing $0.1 \%$ collagenase (collagenase type I; Worthington Biochemical Corporation, Lakewood, NJ, USA). The digests were filtered through sterile gauze and centrifuged at $210 \times g$ for $5 \mathrm{~min}$ at room temperature. After removing the resulting supernatant, the SVF was resuspended in cell culture medium (cell-CM) consisting of Dulbecco's modified Eagle's medium (Life Technologies, Carlsbad, CA, USA), 10\% fetal bovine serum (FBS; HyClone: Cytiva,
Tokyo, Japan), and $1 \%$ antibiotics $(100 \mathrm{U} / \mathrm{mL}$ penicillin, $100 \mu \mathrm{g} / \mathrm{mL}$ streptomycin, and $25 \mu \mathrm{g} / \mathrm{mL}$ amphotericin B; Antibiotic-Antimycotic, Life Technologies). The cells were centrifuged a second time, resuspended in fresh cell$\mathrm{CM}$, and seeded in $75 \mathrm{~cm}^{2}$ culture flasks (Iwaki, Shizuoka, Japan). Following incubation at $37{ }^{\circ} \mathrm{C}$ and $5 \% \mathrm{CO}_{2}$ for 7 days, adherent cells were washed with PBS and cultured in fresh cell-CM as passage 0. Seven days after seeding, the cells were harvested with recombinant trypsin (TrypLE Select; Life Technologies), diluted with an equal volume of fresh cell-CM, and centrifuged. After discarding the supernatant, the pellet was rinsed with fresh cell-CM, seeded at $1.0 \times 10^{6}$ cells per $15-\mathrm{cm}$ dish (Falcon; Corning Inc., Corning, NY, USA), and cultured until $90 \%$ confluence was achieved. This process was repeated once more (until passage 2) to obtain a sufficient number of cells for generating a scaffold-free 3D ADSC construct (Fig. 1B). For all cell culturing, the medium was changed every 2 days.

\subsection{Trilineage differentiation}

ADSCs collected from the subcutaneous adipose tissue of the intrascapular region of rats were differentiated into adipocytes, chondrocytes, and osteocytes (Fig. 1C).

\subsubsection{Adipogenic differentiation of ADSCs}

The collected ADSCs were seeded at $2.0 \times 10^{5}$ cells per well in 6-well plates (Nunc Cell-Culture Treated Multidishes; Thermo Fisher Scientific, Waltham, MA, USA) and cultured in cell-CM at $37{ }^{\circ} \mathrm{C}$ and $5 \% \mathrm{CO}_{2}$ until reaching $90 \%$ confluence. The cell-CM was then changed to adipogenic induction medium (Lonza, Walkersville, MD, USA), consisting of $4.5 \mathrm{~g} / \mathrm{L}$ D-glucose, 100 $\mu \mathrm{M}$ indomethacin, $10 \mu \mathrm{g} / \mathrm{mL}$ insulin, $0.5 \mathrm{mM}$ 3-isobutyl1-methylxanthine, and $1 \mu \mathrm{M}$ dexamethasone, and cultured for another 6 days. The cells were then formalin-fixed and 
stained with Oil red O (Muto Pure Chemicals, Co., Ltd., Tokyo, Japan) to assess the production of oil droplets. Cells cultured in cell-CM for 6 days were used as controls. The medium was changed every 3 days.

\subsubsection{Chondrogenic differentiation of ADSC spheroids}

Cell suspensions of $2.5 \times 10^{4}$ ADSCs were prepared in 15-mL tubes (Falcon; Corning Inc.) and centrifuged at $210 \times g$ for $5 \mathrm{~min}$ at room temperature, after which the supernatant was removed. Subsequently, ADSCs were suspended in $1 \mathrm{~mL}$ of chondrogenic induction medium (CIM; Differentiation Basal Medium-Chondrogenic, Lonza) containing $4.5 \mathrm{~g} / \mathrm{L}$ D-glucose, $350 \mu \mathrm{M}$ L-proline, $100 \mathrm{nM}$ dexamethasone, $10 \mathrm{ng} / \mathrm{mL}$ transforming growth factor beta 3 (TGF- $\beta 3$ ), and $500 \mathrm{ng} / \mathrm{mL}$ bone morphogenetic protein (BMP)-2, followed by centrifugation at $210 \times \mathrm{g}$ for $5 \mathrm{~min}$ at room temperature and incubation at $37{ }^{\circ} \mathrm{C}$ and $5 \% \mathrm{CO}_{2}$ for 28 days. The medium was changed every 3 days. After induction, frozen sections ( $7 \mu \mathrm{m}$ thick) (Leica CM1950; Leica Biosystems, Nussloch, Germany) were prepared following formalin fixation and then stained with hematoxylin and eosin (HE; Muto Pure Chemicals, Co., Ltd.), Alcian blue (Fujifilm Wako Pure Chemical Industries, Ltd.), and Safranin O (Muto Pure Chemicals, Co., Ltd.). Cells cultured in construct culture medium [construct-CM: 1:1 mixture of endothelial cell growth medium (EGMTM-2 BulletKit ${ }^{\mathrm{TM}}$, Lonza) and fibroblast growth medium (FGM ${ }^{\mathrm{TM}}$ 2 BulletKit ${ }^{\mathrm{TM}}$, Lonza)] for 28 days were used as controls. Endothelial cell growth medium is comprised of endothelial basal medium (Lonza) containing vascular endothelial growth factor, human epidermal growth factor, arginine 3-insulin-like growth factor-1, ascorbic acid, hydrocortisone, basic human fibroblast growth factor- $\beta$, heparin, FBS, and GA-1000 (gentamicin and amphotericin B). Fibroblast growth medium is comprised of fibroblast basal medium (Lonza) containing insulin, recombinant human basic fibroblast growth factor, GA-100, and FBS. The concentrations of the additive factors for endothelial cell and fibroblast growth media are not known because they were not disclosed by the manufacturer.

\subsubsection{Osteogenic differentiation of ADSCs}

For osteogenic differentiation, ADSCs were seeded in a 6-well plate (Thermo Fisher Scientific) with cell$\mathrm{CM}$ at an initial density of $3.0 \times 10^{4}$ cells/well. At $80 \%$ confluence, the medium was replaced with osteogenic induction medium (OIM; Differentiation Basal MediumOsteogenic, Lonza) containing $100 \mu \mathrm{M}$ ascorbic acid, 10 $\mathrm{mM} \beta$-glycerophosphate, and $1 \mu \mathrm{M}$ dexamethasone, and cultured for 28 days. The medium was changed every 3 days. After formalin fixation, the samples were stained with Alizarin red (Wako Pure Chemical Industries, Ltd., Osaka, Japan) to assess calcium deposition. Cells cultured in 6well plates with cell-CM for 28 days were used as controls.

\subsubsection{Osteogenic differentiation of ADSC spheroids}

Two cell suspensions of $2.5 \times 10^{4}$ ADSCs in $15-\mathrm{mL}$ tubes (Falcon ${ }^{\circledR}$; Corning Inc.) were centrifuged at $210 \times g$ for $5 \mathrm{~min}$ at room temperature, and then the supernatants were discarded. Subsequently, OIM with and without additional factors (500 ng/mL BMP-2, $10 \mathrm{ng} / \mathrm{mL}$ TGF- $\beta 3$ ) were prepared, and ADSCs were suspended in the respective media. The cells were then centrifuged at $210 \times g$ for $5 \mathrm{~min}$ at room temperature to form a spheroid and incubated at $37^{\circ} \mathrm{C}$ and $5 \% \mathrm{CO}_{2}$ for 28 days; the medium was changed every 3 days. Micro-computed tomography $(\mu \mathrm{CT})$ imaging (described below) was performed after incubation, followed by formalin fixation and staining of frozen sections (7 $\mu \mathrm{m}$ thick) with Alizarin red and von Kossa stain. Spheroids incubated with the cell-CM construct for 28 days were used as controls.

\subsection{Fabrication of a scaffold-free 3D ADSC construct}

At least $9.6 \times 10^{6}$ cells were used to fabricate each construct with a bio-3D printer (Regenova; Cyfuse Biomedical K.K., Tokyo, Japan) and a needle array (hollow $9 \times 9$ Kenzan; Cyfuse Biomedical K.K.) (Fig. 2). Briefly, cells $\left(3.0 \times 10^{4}\right.$ cells/well $)$ were inoculated into four $96-$ well non-adhesion plates (PrimeSurface 96U Plate; Sumitomo Bakelite, Tokyo, Japan) and cultured in construct-CM. After incubation for $48 \mathrm{~h}$, the cells formed spheroids with diameters of approximately $550 \mu \mathrm{m}$. The spheroids were then arranged on a Kenzan using the bio-3D printer and printed according to a pre-made design using B3D designer software (Cyfuse Biomedical K.K.; Fig. 1F), as previously reported [35]. The process took approximately $1.5 \mathrm{~h}$.
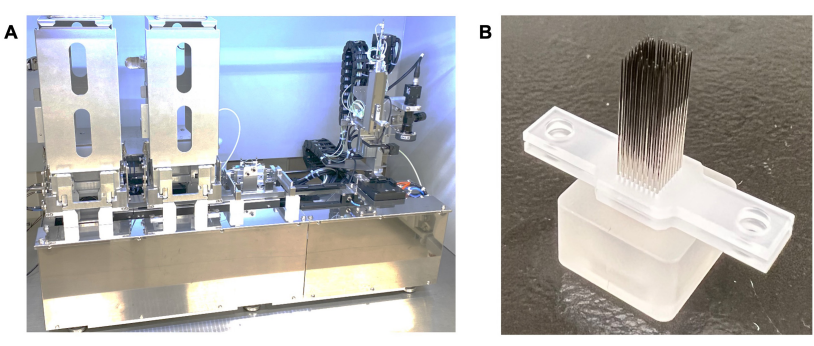

Fig. 2. Bio-3D printer with a needle array (Kenzan). (A) Bio3D printer. (B) Hollow $9 \times 9$ needle array (Kenzan).

\subsection{Maturation of the ADSC construct}

The Kenzan was placed in an original perfusion chamber, which can directly supply the hollow structure with medium, and then cultured with the spheroids at $37^{\circ} \mathrm{C}$ and $5 \% \mathrm{CO}_{2}$ for 5 days to fuse them together. On the fifth day after printing, a construct with a diameter of $3 \mathrm{~mm}$ and height of $3 \mathrm{~mm}$ was obtained and removed from the Kenzan. Another construct was also obtained through the same process above, the two constructs were placed side by side 
on a plastic tube to allow them to fuse together (Fig. 3), and then they were cultured in construct-CM for 10 days. Thus, the total culture period was 15 days, including 5 days on Kenzan and 10 days on the plastic tube. Subsequently, a connected ADSC construct was subjected to a tensile test.

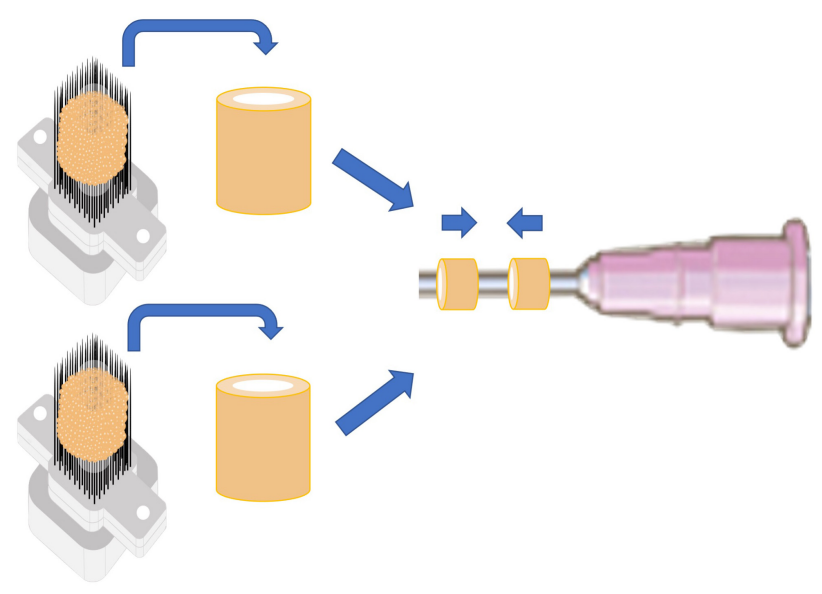

Fig. 3. Fusion of two ADSC constructs. Two constructs were cultured side by side on a plastic tube to facilitate fusion.

\subsection{Mechanical properties of the ADSC constructs}

Tensile tests were performed using a Tissue Puller ${ }^{\mathrm{TM}}$ device (DMT, Ann Arbor, MI, USA) for three fused ADSC constructs. Small stainless pins served as grips for the individual structures, after which the constructs were pulled in tension to failure at a rate of $50 \mu \mathrm{m} / \mathrm{s}$ (Supplementary data 1). The load $(\mathrm{mN})$ was defined as the load applied to the construct at the time of rupture; the strength was calculated as the load per unit area by calculating the contact area between two pins: load $(\mathrm{mN}) /[$ width of construct $(\mathrm{mm})$ $\times$ thickness of construct $(\mathrm{mm}) \times 2$ ], and the stiffness was calculated as the load per unit length based on the degree of extension until the construct ruptured: load $(\mathrm{mN}) /$ extension of the construct to the point of rupture $(\mathrm{mm})$.

\subsection{Fabrication of a scaffold-free chondrogenic-induced construct}

The prepared ADSC constructs were divided into a chondrogenic induction group and a control group. The induction group comprised two subgroups: one in which induction with CIM was started immediately after printing and another in which induction was started when the construct was removed from the Kenzan 4 days after printing. The control group was cultured in construct-CM. Culturing was performed in the original bioreactor at $37{ }^{\circ} \mathrm{C}$ and $5 \%$ $\mathrm{CO}_{2}$ for 28 days at a perfusion flow rate of approximately $6 \mathrm{~mL} / \mathrm{min}$. The culture medium was changed every 3 days.

\subsection{Fabrication of a scaffold-free osteogenic-induced construct}

The prepared ADSC constructs were divided into an osteogenic induction group and a control group. The induction group comprised two subgroups: one in which induction with OIM was started immediately after printing and another in which induction was started when the construct was removed from the Kenzan 4 days after printing. The OIM was supplemented with additional factors $(10 \mathrm{ng} / \mathrm{mL}$ TGF- $\beta 3,500 \mathrm{ng} / \mathrm{mL}$ BMP-2, and $2.4 \mathrm{mM}$ PBS, $\mathrm{pH}$ 7.4). The cultures were perfused in the original perfusion chamber at $37{ }^{\circ} \mathrm{C}$ and $5 \% \mathrm{CO}_{2}$ at a flow rate of approximately $6 \mathrm{~mL} / \mathrm{min}$. After 14 days of culture in OIM, $1 \mu \mathrm{g} / \mathrm{mL}$ of calcitonin and $1 \mu \mathrm{g} / \mathrm{mL}$ of insulin-like growth factor-1 were added to both induction subgroups [36], which were then cultured for a further 14 days. The control group was cultured in construct-CM for 28 days. The medium was changed every 3 days.

\section{$2.9 \mu C T$}

The fabricated spheroids and constructs were imaged in 96-well plates using $\mu \mathrm{CT}$ (3D Micro X-ray CT Lab GX130; Rigaku Corporation, Yamanashi, Japan) with the following parameters: tube voltage, $90 \mathrm{kV}$; tube current, 61 $\mu \mathrm{A}$; field of view, $72 \mathrm{~mm}$; and high resolution $(9 \mu \mathrm{m} / \mathrm{pixel})$. The images were taken at a speed of one revolution around the sample in $4 \mathrm{~min}$. A rat femur cut into 5-mm lengths and uninduced control constructs were used as controls. For the spheroid, 3D images were acquired only for structures with CT values greater than or equal to the CT value that clearly showed only the rat femur fragment $(\mathrm{CT}$ value $=775$ Hounsfield Unit (HU)). The 3D images of the constructs were acquired in the same way $(\mathrm{CT}$ value $=1069 \mathrm{HU})$, and the $\mathrm{CT}$ values of the depicted structures were determined by setting the lower limit for obtaining 3D images in the CT Lab GX130 system used for imaging (CT values of the bones ranged from 100 to $1000 \mathrm{HU}$ ). The $\mathrm{CT}$ values were acquired with the calibration settings of $-1000 \mathrm{HU}$ for air and $20 \mathrm{HU}$ for acrylic plate according to the instructions of CT Lab GX130.

\subsection{Histological analysis of the ADSC constructs and the chondrogenic-and osteogenic-induced constructs}

Constructs were fixed in 10\% neutral-buffered formalin for 15 days and embedded in paraffin. Serial sections ( $7 \mu \mathrm{m}$ thick) were then obtained and placed on glass slides for staining with HE, Alcian blue, Safranin O, Alizarin red, and von Kossa stains. Histological sections were prepared and stained by Advantec Co., Ltd. (Tokyo, Japan).

\section{Results}

\subsection{Trilineage differentiation of ADSCs}

Oil red $\mathrm{O}$ staining of the adipogenic-induced ADSCs showed reddish oil droplets in a greatly expanded cytoplasm (Fig. 4A). The cells differentiated very quickly 
A

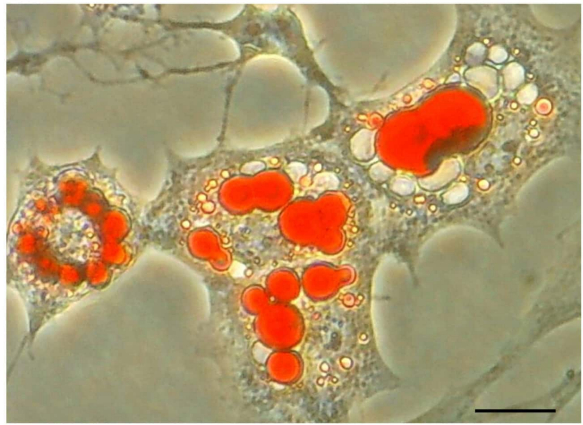

C

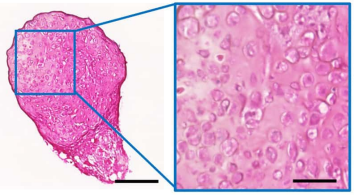

F

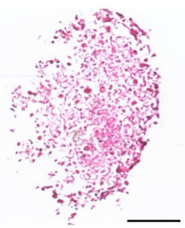

D

H
B
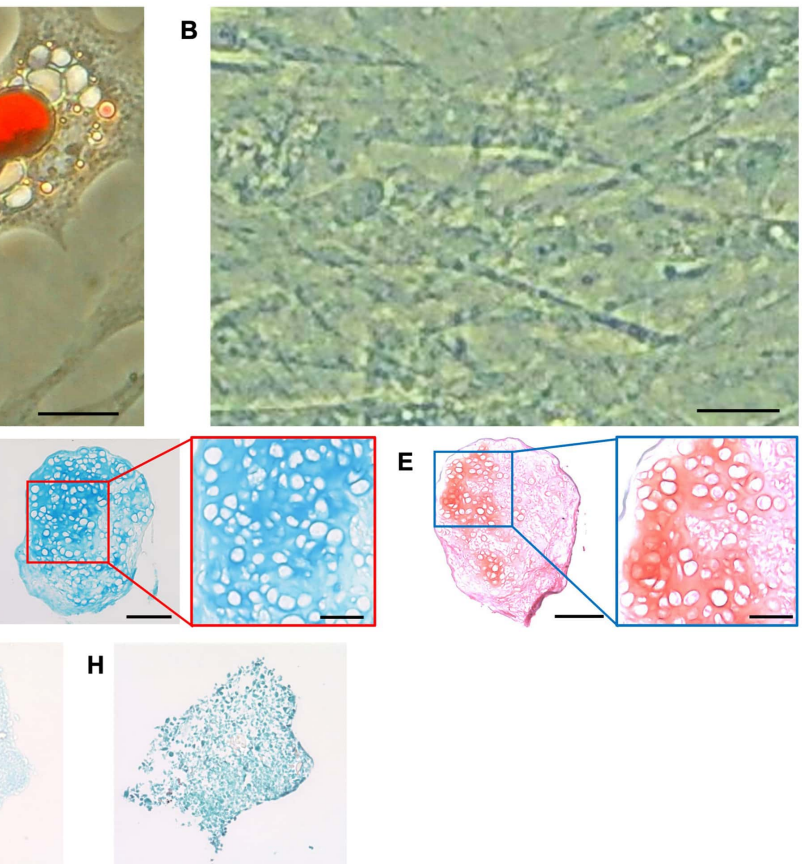
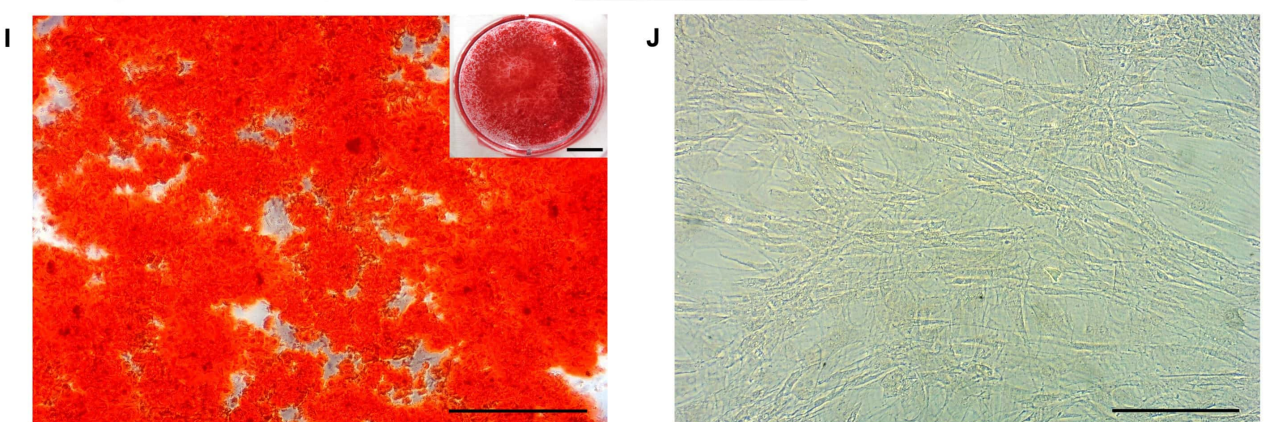

K

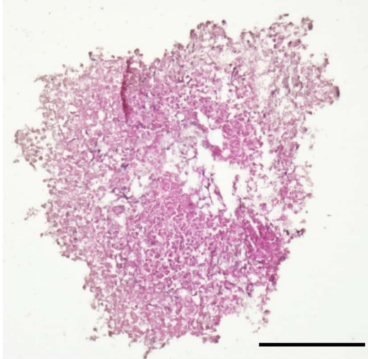

N

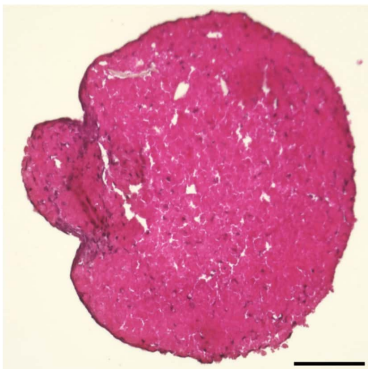

L

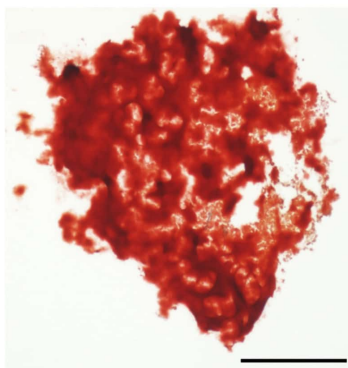

$\mathbf{0}$

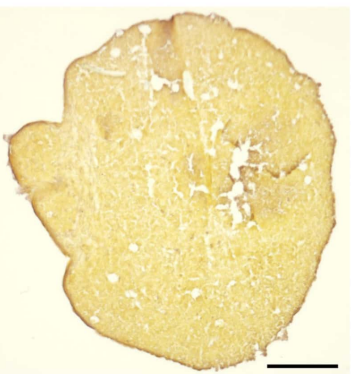

M

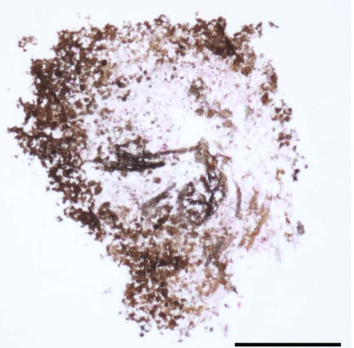

$\mathbf{P}$

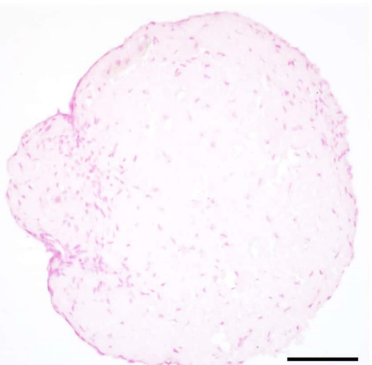

Fig. 4. Adipogenic, chondrogenic, and osteogenic differentiation of ADSCs. (A,B) Oil red O staining of induced (A) and uninduced control (B) ADSCs. Scale bar $=50 \mu \mathrm{m}$. (C-H) Chondrogenic differentiation of ADSC spheroids. HE, Alcian blue, and Safranin O staining of induced (C-E) and uninduced control (F-H) spheroids. Scale bar $=200 \mu \mathrm{m}$ and $100 \mu \mathrm{m}$ (magnified image). (I-P) Osteogenic differentiation of ADSCs. Alizarin red staining of induced (I) and uninduced control (J) ADSCs. Scale bar $=200 \mu \mathrm{m}$ and $1 \mathrm{~cm}$ (for the whole image). (K-P) Osteogenic differentiation of ADSC spheroids. HE, Alizarin red, and von Kossa staining of induced (K-M) and uninduced control (N-P) spheroids. Scale bar $=200 \mu \mathrm{m}(\mathrm{K}-\mathrm{M})$ and $100 \mu \mathrm{m}(\mathrm{N}-\mathrm{P})$. 


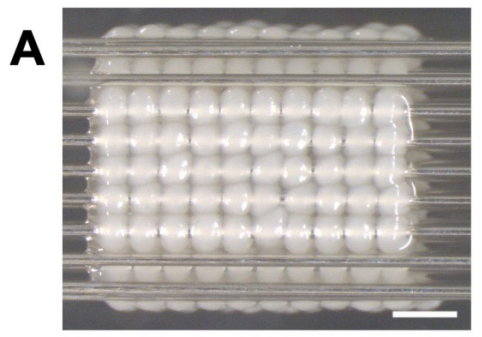

day 0

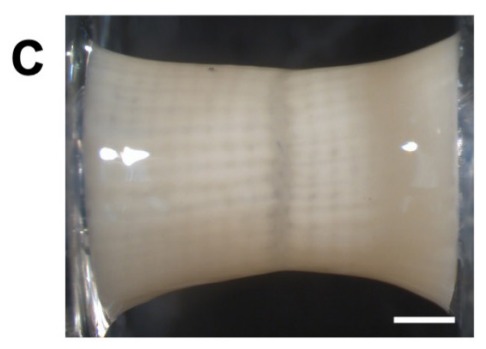

day5

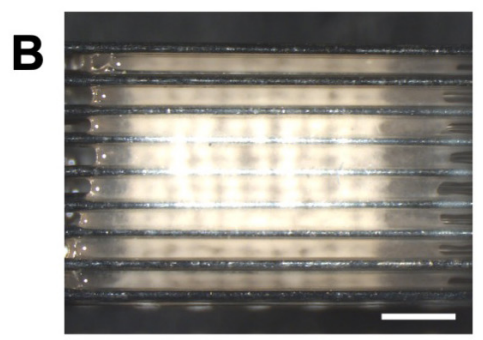

day5

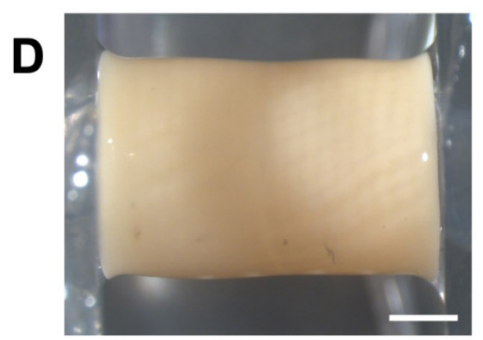

day 15

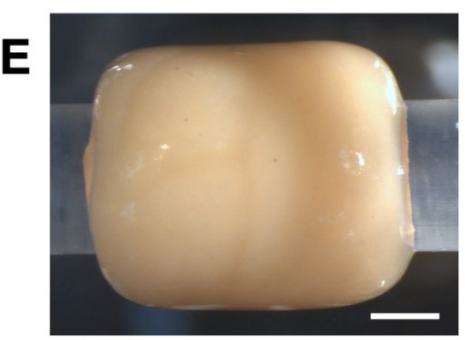

day28

Fig. 5. Fabrication of fused ADSC constructs. (A) The spheroids were arranged on the needles of the Kenzan as per the computer design (day 0). (B) After 5 days of incubation, the spheroids fused together to form a single construct (day 5). (C) The two constructs were removed from the Kenzan and placed side by side on a plastic tube (day 5). (D) Two constructs fused together in maturation culture (day 15; 5 days on Kenzan and 10 days on the plastic tube). (E) Constructs that have undergone further incubation in the maturation culture (day 28; 5 days on Kenzan and 23 days on the plastic tube). Scale bar $=1 \mathrm{~mm}$.

(within 6 days) and produced oil droplets. The production of oil droplets indicates that the ADSCs differentiated into adipocytes. In uninduced ADSCs, no oil droplet production was observed and staining was negative (Fig. 4B).

After chondrogenic induction of ADSC spheroids, large oval cells were observed with an Alcian blue- and Safranin O-stained cartilage matrix surrounding the cells (Fig. 4C-E). In contrast, the uninduced spheroids showed no large oval cells and the staining was negative (Fig. 4F$\mathrm{H})$.

For osteogenic induction of ADSCs, the entire basal surface of the well surrounding the cells was strongly stained with Alizarin red (Fig. 4I), whereas uninduced ADSCs were negative for Alizarin red staining (Fig. 4J). After osteogenic induction and staining of spheroids, whole spheroids were found to be positively stained with Alizarin red (Fig. 4L), while von Kossa staining confirmed the presence of calcium throughout the spheroid (Fig. 4M); in contrast, the uninduced spheroids were negative for Alizarin red and von Kossa staining (Fig. 4O,P).

\subsection{Tensile strength of the ADSC constructs}

The load, strength, and stiffness of the three fused ADSC constructs were measured (Fig. 5). The calculated values were as follows: load, $195.3 \pm 6.1 \mathrm{mN}$ (Supplementary data 2-4); strength, $39.1 \pm 1.2 \mathrm{kPa}$; and stiffness, $0.09 \pm 0.01 \mathrm{~N} / \mathrm{mm}$. The fabricated constructs had the strength to withstand these values. However, as shown in the supplemental data, there are slight individual differences in the ADSC constructs, which may result in non-uniform tensile loading. Therefore, the values obtained from these measurements were used only as estimates for the strength evaluation of the constructs.

\section{$3.3 \mu$ CT of osteogenic-induced spheroids}

Osteogenic-induced spheroids were subjected to $\mu \mathrm{CT}$ imaging. Only the spheroids induced in OIM with additional factors showed CT values $(\mathrm{CT}$ value $=775 \mathrm{HU}) \mathrm{com}$ parable to those of bone (The range of CT values for bone is 100-1000 HU [37].) (Fig. 6B-iii). Spheroids induced only by OIM were visible when the lower limit of the CT value was set to 353 but became invisible when the lower limit of the CT value was increased to 775 (Fig. 6A-ii).

\section{$3.4 \mu C T$ of osteogenic-induced constructs}

The two induced and control constructs were subjected to $\mu \mathrm{CT}$ imaging analysis (Fig. 7A,B). They showed comparable CT values to those of bone (CT value $=1069$ $\mathrm{HU})$. Calcification was detected for both groups of induced constructs; however, when the CT value was adjusted to be around 1000, which is the CT value of bone, it was not depicted in the Day 0 constructs. In the case of the day 4 constructs, calcification was observed, but only a part of the constructs exhibited CT values comparable to those of the rat femur (Fig. 7C,D). 
A

(i)

(ii)

(iii)

(iv)

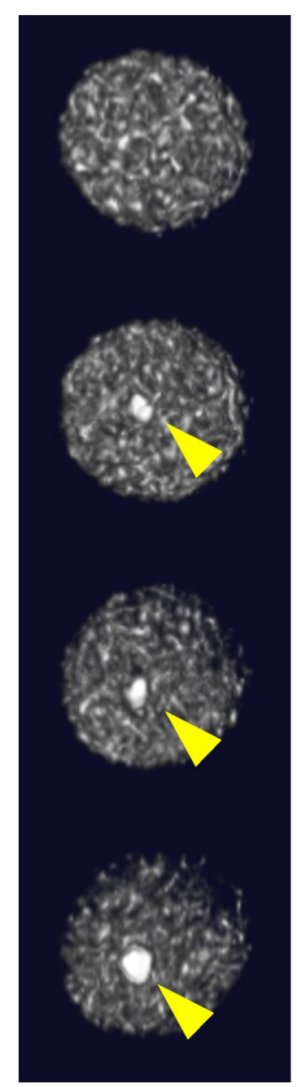

B

(i)

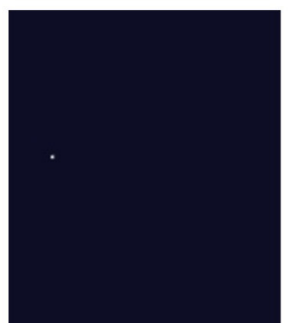

(ii)

(iii)

(iv)

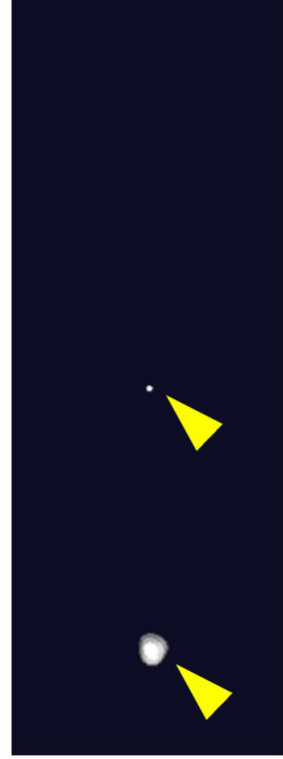

Fig. 6. $\mu$ CT images of osteogenic-induced spheroids. (A) Widewindow level 3D CT image. The lower limit of the CT value used for $3 \mathrm{D}$ image construction was $353 \mathrm{HU}$. (B) Adjusted window level to the cortical bone. The lower limit of the CT value used for 3D image construction was 775 HU. (i) Uninduced spheroid. (ii) Induced with only OIM. (iii) Induced spheroid with OIM supplemented with BMP-2 and TGF- $\beta 3$. (iv) Finely ground rat femur bone fragment. The yellow arrowheads indicate spheroids (A-ii, A-iii, B-iii) and the bone fragment (A-iv, B-iv).

\subsection{Histology of the ADSC constructs}

HE staining of the uninduced constructs showed that the boundaries between the spheroids were blurred and that the spheroids were fused into a single construct. In addition, nuclei were found distributed throughout the structure, confirming that the cells were evenly distributed (Fig. 8A). Alcian blue, Alizarin red, von Kossa, and Safranin O staining were negative, and no cartilage matrix was observed (Fig. 8B-E).

\subsection{Histology of the chondrogenic-induced constructs}

For both the day 0 and day 4 chondrogenic-induced constructs, large oval cells were observed whose nuclei were strongly stained with HE, similar to the results of the chondrogenic-induced spheroids (Fig. 8F,K). Alcian blue staining indicated the presence of an abundant cartilage matrix around the cells (Fig. 8G,L), which was also positively

stained with Safranin O (Fig. 8H,M). The constructs were negative for Alizarin red and von Kossa staining, and no calcium production was observed (Fig. 8I,J,N,O). In the day 0 construct, chondrogenic differentiation was uniformly observed along the periphery of the constructs, and a layered structure was formed (Fig. 8F,G,H). In contrast, the day 4 structures showed no regularity in the areas of chondrogenic differentiation and fewer areas were stained more darkly with Alcian blue and Safranin O compared with the staining of the day 0 construct (Fig. 8L,M). Thus, the day 0 construct showed more pronounced chondrogenic differentiation than the day 4 construct. In common with both the day 0 and day 4 structures, chondrogenic differentiation was relatively poor inside the structures in contact with the plastic tube.

\subsection{Histology of the osteogenic-induced constructs}

Histological specimens of the osteogenic-induced constructs showed areas that were simultaneously stained with HE, Alizarin red, and von Kossa, indicating calcium deposition (Fig. 8P,S,T,U,X,Y). In contrast, Alcian blue and Safranin O staining were negative (Fig. 8Q,R,V,W), and no cartilage matrix was observed. There was no substantial difference noted in the areas stained with Alizarin red and von Kossa stain between the day 0 and day 4 constructs. With respect to the distribution of calcified areas, most of the intensely stained areas in the day 0 constructs were found inside the constructs, whereas most of these areas were observed at the periphery of the day 4 constructs.

\section{Discussion}

In this study, we successfully fabricated the first scaffold-free bone-like constructs and cartilage-like constructs using rat ADSCs. In particular, reconstruction of the bone tissue with sufficient strength in vitro has not been achieved to date. Therefore, we believe that one effective approach would be to implant our scaffold-free ADSC constructs in an uninduced state and allow them to mature into bone tissue in vivo. Furthermore, we confirmed that the two constructs could fuse together. By fusing constructs, the size and shape of the constructs that can be fabricated becomes more diverse. The results of the trilineage differentiation experiments indicated that ADSCs have the ability to differentiate not only into adipose tissue but also into bone and cartilage.

The strength test showed similar results $(39.1 \pm 1.2$ $\mathrm{kPa}$ ) for all three fused constructs, indicating that they were fabricated with comparable quality. According to a previous report [38], the ADSC constructs can be considered to be as strong as the muscle tissue and thyroid gland based on the estimated strength measured in this study. Unfortunately, we were unable to induce sufficient calcification of the constructs in this study; therefore, we could not test the strength of the osteogenic-induced structures with a compression test, which is considered to be the most appropriate 
A

C (i) (ii)

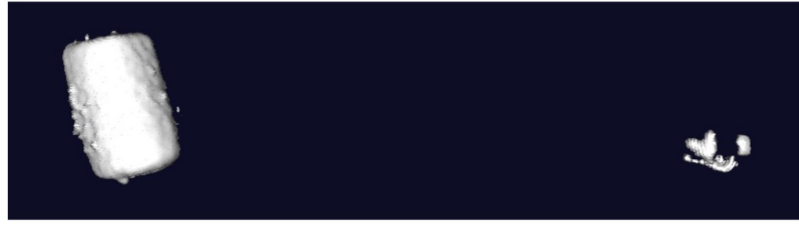

B

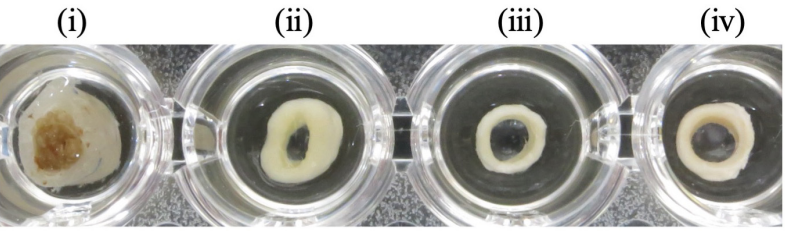

D

(i)

(ii)

(iii)

(iv)

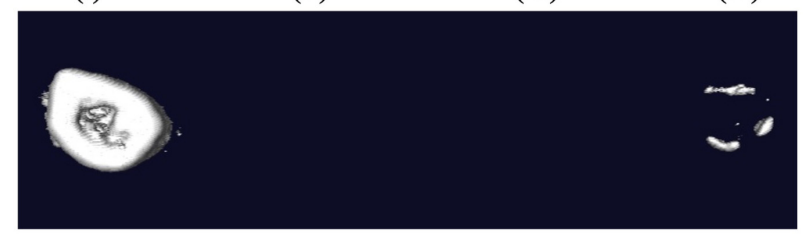

Fig. 7. Macroscopic and $\mu$ CT images. (A) Side view. (B) Top view. (C) Side view. (D) Top view. (i) A part of the rat femur. (ii) Uninduced construct. Construct induced on day 0 (iii) and day 4 (iv) after printing. The lower limit of the CT value used for 3D image construction was $1069 \mathrm{HU}$.
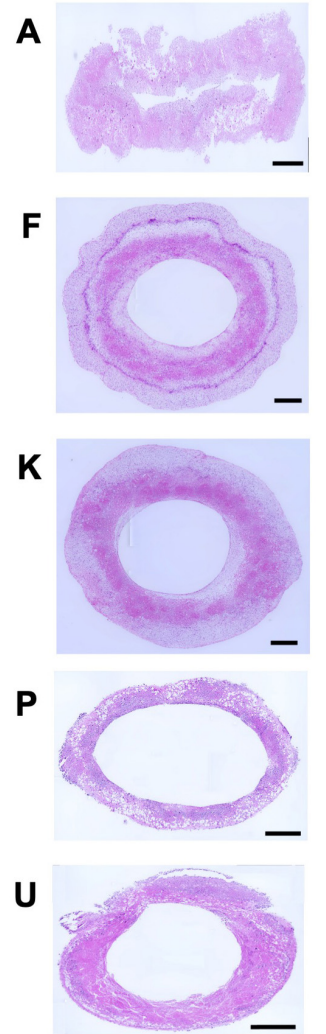

B

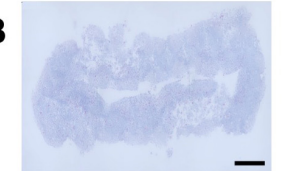

G

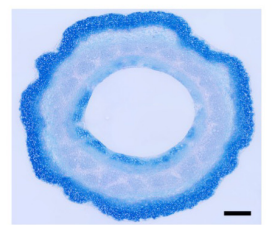

$\mathbf{L}$

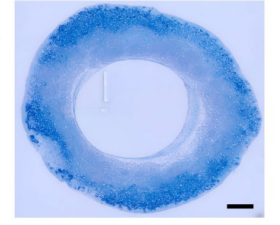

$\mathbf{Q}$

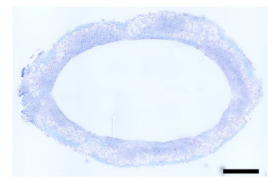

V

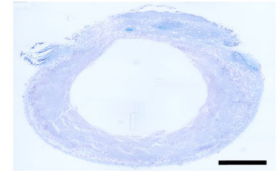

C

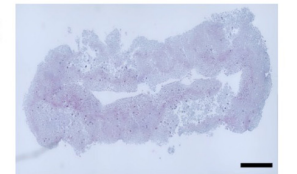

$\mathbf{H}$

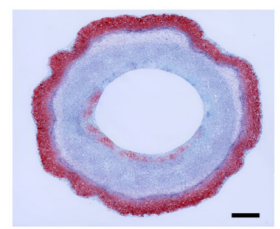

M

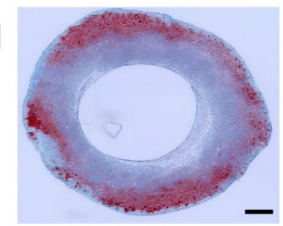

$\mathbf{R}$

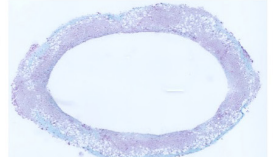

w

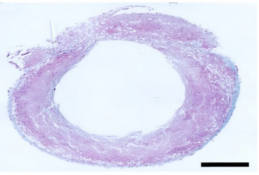

D

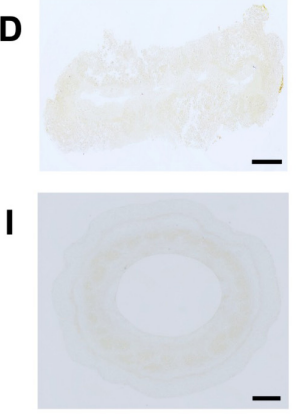

$\mathbf{N}$

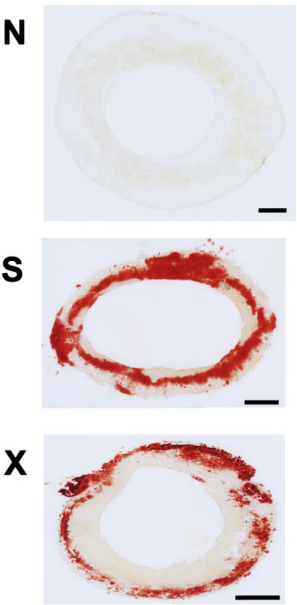

E

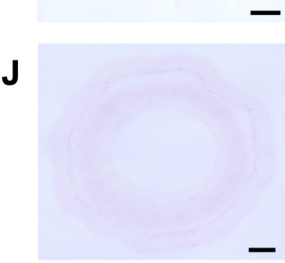

o
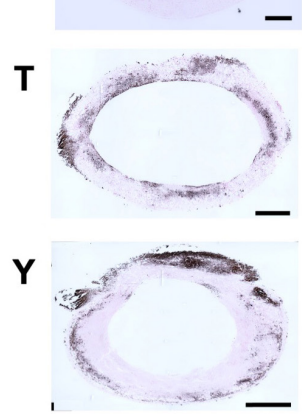

Fig. 8. Histology of the uninduced and induced constructs. (A-E) HE, Alcian blue, Safranin O, Alizarin red, and von Kossa staining of the uninduced constructs. HE, Alcian blue, Safranin O, Alizarin red, and von Kossa staining of the day 0 (F-J) and day 4 (K-O) chondrogenic-induced constructs. HE, Alcian blue, Safranin O, Alizarin red, and von Kossa staining of the day 0 (P-T) and day 4 (U-Y) osteogenic-induced constructs. Scale bar $=500 \mu \mathrm{m}$.

test given the mechanical properties of bone. However, if a certain level of strength is not obtained in a compression test, the results will be too noisy for accurate interpretation. Therefore, compression tests could not be performed in this study. However, based on our results, we consider that the induced constructs are at least as strong as the uninduced constructs.
Notably, there was a marked difference in the $\mu \mathrm{CT}$ imaging results in the osteogenic induction experiments. The results of $\mu \mathrm{CT}$ imaging of osteogenic-induced spheroids suggest that during the osteogenic induction of ADSCs, TGF- $\beta 3$, BMP-2, and phosphates are required to produce calcification that is comparable to that of the cortical bone. Furthermore, the $\mu \mathrm{CT}$ results of the osteogenic- 
induced constructs suggested that not only the presence of additional factors but also the timing of the initiation of induction had a significant effect on calcification. Bone tissue that has undergone normal development is formed by the deposition of calcium phosphate on type I collagen fibers arranged in a certain direction $[39,40]$. The difference in calcification between the day 0 and day 4 constructs may be due to the extracellular matrix (ECM) component, which includes type I collagen. In the day 4 constructs, sufficient ECM production and the generation of oriented type I collagen fibers prior to calcium salt deposition occurred. Subsequent deposition of calcium salts along the oriented collagen fibers is thought to have resulted in the construction of a dense, regularly aligned crystal structure. In the day 0 constructs, ECM production and calcium salt deposition occurred simultaneously, and the type I collagen fiber orientation did not form; consequently, dense calcified structures such as cortical bone did not form, and the constructs may not have been visible on CT. Thus, to efficiently and densely retain the calcium secreted by osteoblasts in the constructs, it is necessary to optimize the timing of the initiation of osteogenesis induction and to further investigate approaches for regulating the production and orientation of the ECM.

Comparison of the histological evaluation of uninduced, chondrogenic-induced, and osteogenic-induced constructs showed that ADSCs maintain their differentiation potential after forming constructs. Chondrogenicinduced constructs showed more pronounced chondrogenic differentiation at day 0 compared with that of the day 4 constructs. The cartilage matrix is produced by chondrocytes and is rich in type II collagen fibers [41]. It is presumed that chondrogenic differentiation is promoted by starting the induction process immediately after printing, which results in the production of a large amount of ECM around the cells, as a characteristic of cartilage tissue such as type II collagen fibers. It is possible that during spheroid fusionbefore induction - of the day 4 constructs, cellular adhesion was instead enhanced, and the ECM components specific to the cartilage tissue, such as proteoglycans, could not be produced around the cells, or other types of collagen fibers such as type I collagen, which is not specific to cartilage tissue, were produced. This is consistent with our previous study on cartilage constructs using induced pluripotent stem cells (iPSCs), which showed that the timing of additive factors for induction is very important [41]. The scaffold-free ADSC constructs prepared in this study were found to be as strong as soft tissues such as the muscle tissue and thyroid gland in the uninduced state, and we assume that the induced constructs have the same or greater strength. To our knowledge, there are no reports of scaffold-free 3D cartilage constructs fabricated using only ADSCs, which have advantages over smaller constructs such as micromasses or spheroids. This is one of the main new achievements in this study. In our previous study, we found that both bone and cartilage were regenerated after implantation of ADSC constructs into osteochondral defects in the knee joints [42,43]. Another report demonstrated the formation of hyaline cartilage after subcutaneous implantation of fabricated cartilage pellets, although these were derived from iPSCs [44]. Our cartilage-like construct is expected to have application in the reconstruction of bone defects through the process of endochondral ossification after implantation.

Histological evaluation of the osteogenic-induced constructs showed strong staining with Alizarin Red and von Kossa, which was inconsistent with the results of CT imaging, indicating that calcium salt deposition by tissue staining does not correlate with $\mathrm{CT}$ imaging results. For histological evaluation of bone-like constructs, the presence of calcification alone is not sufficient. However, if the construct can be detected in CT images, it can be assumed that the crystal structure is at least sufficiently dense to not be penetrated by X-rays. There have been several reports on the fabrication of bone-like constructs using scaffolds $[36,45,46]$; however, producing calcified scaffold-free 3D constructs with CT values comparable to those of normal cortical bone in vitro is unprecedented. Furthermore, there is no previous description of the strength of bone-like tissue constructs fabricated using existing scaffolds, and it remains questionable whether structures with strength comparable to that of bone tissue can be fabricated. Based on existing reports, the fabrication of bone-like constructs in vitro remains a very challenging task. Moreover, we were unable to perform compression tests in this study because we could not achieve calcification of the entire structure; nevertheless, the fact that we were able to produce calcified $3 \mathrm{D}$ constructs with $\mathrm{CT}$ values comparable to cortical bone using only ADSCs is considered to be a major achievement. The final goal of this study was to develop an alternative clinical technique for $\mathrm{AB}$ implantation. For this purpose, it was necessary to fabricate strong constructs. It has been reported that osteogenesis of the bone tissue is accelerated by mechanical stimulation [47]; however, we did not mechanically stimulate the constructs, as we only employed perfusion culture. It is thus possible that the lack of mechanical stimulation may have contributed to the partial calcification and insufficient construct strength. In the future, the effects of incubation with mechanical loading should also be investigated.

There are also reports of improved wound healing [31,32] and accelerated angiogenesis [33] when ADSCs were used in combination with hyaluronic acid or PRP; thus, these biological materials are expected to be applied to the fabrication of similar new constructs for applications in various fields other than bone and cartilage regeneration. Finally, it is possible that human ADSCs would behave differently from rat ADSCs under the same experimental conditions adopted in this study; therefore, it is necessary to study larger animals and cells from animal species that are more similar to humans. In addition, the larger the animal, 
the larger the constructs that need to be fabricated. As the calcification of larger construct is expected to be more difficult from the viewpoint of nutrient supply, improvement of the method of construct preparation and culture for such scale-up remains an important issue for future investigation.

\section{Conclusions}

In this study, we fabricated scaffold-free bone- and cartilage-like constructs consisting of ADSCs without AMs. This is the first study to show that scaffold-free AD$\mathrm{SCs}$ can maintain their $3 \mathrm{D}$ structure and produce the same CT values as the cortical bone without the use of scaffolds or any AMs. Moreover, the chondrogenic-induced constructs are expected to differentiate into bone tissue through endochondral ossification after implantation, and we believe that they can also be applied for cartilage regeneration by ADSCs, similar to our previous findings on cartilage regeneration using iPSCs [41]. As there are no previous reports of scaffold-free bone-like and cartilage-like constructs using ADSCs in vitro, our results highlight the potential of ADSCs for bone and cartilage reconstruction.

\section{Abbreviations}

$\mu \mathrm{CT}$, micro-computed tomography; 3D, threedimensional; AB, autogenous bone; ADSC, adiposederived stromal cell; AM, artificial material; BMP, bone morphogenic protein; CM, culture medium; CIM, chondrogenic induction medium; ECM, extracellular matrix; EBD, extensive bone defect; FBS, fetal bovine serum; HE, hematoxylin and eosin; iPSC, induced pluripotent stem cell; OIM, osteogenic induction medium; PBS, phosphate-buffered saline; PRP, platelet-rich plasma; SVF, stromal vascular fraction; TGF- $\beta 3$, transforming growth factor beta 3; HU, Hounsfield Unit.

\section{Author contributions}

RF performed the research and prepared the paper. DM designed and initiated the study, prepared the paper, and interpreted the data. $\mathrm{KN}$ conceived and supervised this study. All authors read and approved the final manuscript.

\section{Ethics approval and consent to participate}

All procedures were evaluated and approved by the Institutional Animal Care and Use Committee of Saga University (application no. A2020-048-0).

\section{Acknowledgment}

The authors thank Editage for language editing support and Advantec Co., Ltd. for help with histology.

\section{Funding}

This research was funded by the Japanese Society for the Promotion of Science, grant number 19K18503 (to $\mathrm{DM})$.

\section{Conflict of interest}

$\mathrm{KN}$ is a co-founder and shareholder of Cyfuse Biomedical K.K. as well as an inventor/developer who holds the patent for the Bio-3D printer. Patent title: Method for production of three-dimensional structure of cell; Patent number: JP4517125. Patent title: Cell structure production device; Patent number; JP 5896104. The other authors declare that there is no conflict of interest regarding the publication of this article.

\section{Supplementary material}

Supplementary material associated with this article can be found, in the online version, at https://www.imrpre ss.com/journal/FBL/27/2/10.31083/j.fbl2702052.

\section{References}

[1] Dermience M, Lognay G, Mathieu F, Goyens P. Effects of thirty elements on bone metabolism. Journal of Trace Elements in Medicine and Biology. 2015; 32: 86-106.

[2] Klüter T, Hassan R, Rasch A, Naujokat H, Wang F, Behrendt P, et al. An Ex Vivo Bone Defect Model to Evaluate Bone Substitutes and Associated Bone Regeneration Processes. Tissue Engineering Part C: Methods. 2020; 26: 56-65.

[3] Zekry KM, Yamamoto N, Hayashi K, Takeuchi A, Alkhooly AZA, Abd-Elfattah AS, et al. Reconstruction of intercalary bone defect after resection of malignant bone tumor. Journal of Orthopaedic Surgery. 2019; 27: 230949901983297.

[4] Dimitriou R, Jones E, McGonagle D, Giannoudis PV. Bone regeneration: current concepts and future directions. BMC Medicine. 2011; 9: 66.

[5] Zhao Z, Yan T, Tang X, Guo W, Yang R, Tang S. Novel "doublestrut" fibula ankle arthrodesis for large tumor-related bone defect of distal tibia. BMC Musculoskeletal Disorders. 2019; 20: 367.

[6] Younger EM, Chapman MW. Morbidity at bone graft donor sites. Journal of Orthopaedic Trauma. 1989; 3: 192-195.

[7] Ren Z, Fan T, Zhang S, Wu H. Nonvascularized Iliac Bone Reconstruction for the Mandible without Maxillofacial Skin Scarring. Journal of Oral and Maxillofacial Surgery. 2020; 78: 288294.

[8] Nuri T, Ueda K, Iwanaga H, Otsuki Y, Nakajima Y, Ueno T, et al. Microsurgical mandibular reconstruction using a resin surgical guide combined with a metal reconstructive plate. Microsurgery. 2019; 39: 696-703.

[9] Tosounidis TH, Giannoudis PV. Biological Facet of Segmental Bone Loss Reconstruction. Journal of Orthopaedic Trauma. 2017; 31: S27-S31.

[10] El-Rashidy AA, Roether JA, Harhaus L, Kneser U, Boccaccini AR. Regenerating bone with bioactive glass scaffolds: a review of in vivo studies in bone defect models. Acta Biomaterialia. 2017; 62: 1-28.

[11] Petite H, Viateau V, Bensaïd W, Meunier A, de Pollak C, Bourguignon $\mathrm{M}$, et al. Tissue-engineered bone regeneration. Nature Biotechnology. 2000; 18: 959-963.

[12] Amankwah S, Abdella K, Kassa T. Bacterial biofilm destruction: A focused review on the recent use of phage-based strategies with other antibiofilm agents. Nanotechnology, Science and Applications. 2021; 14: 161-177.

[13] Deng L, Yan Y. Research status and progress of biomaterials for bone repair and reconstruction. Chinese Journal of Reparative and Reconstructive Surgery. 2018; 32: 815-820. (In Chinese)

[14] Kolios G, Moodley Y. Introduction to stem cells and regener- 
ative medicine. Respiration; International Review of Thoracic Diseases. 2013; 85: 3-10.

[15] Shi Y, Inoue H, Wu JC, Yamanaka S. Induced pluripotent stem cell technology: a decade of progress. Nature Reviews. Drug Discovery. 2017; 16: 115-130.

[16] Bunpetch V, Zhang Z, Zhang X, Han S, Zongyou P, Wu H, et al. Strategies for MSC expansion and MSC-based microtissue for bone regeneration. Biomaterials. 2019; 196: 67-79.

[17] Han Y, Li X, Zhang Y, Han Y, Chang F, Ding J. Mesenchymal stem cells for regenerative medicine. Cells. 2019; 8: 886.

[18] Yoshimura H, Muneta T, Nimura A, Yokoyama A, Koga H, Sekiya I. Comparison of rat mesenchymal stem cells derived from bone marrow, synovium, periosteum, adipose tissue, and muscle. Cell and Tissue Research. 2007; 327: 449-462.

[19] Naderi N, Combellack EJ, Griffin M, Sedaghati T, Javed M, Findlay MW, et al. The regenerative role of adipose-derived stem cells (ADSC) in plastic and reconstructive surgery. International Wound Journal. 2017; 14: 112-124.

[20] Zhang J, Liu Y, Chen Y, Yuan L, Liu H, Wang J, et al. AdiposeDerived Stem Cells: Current Applications and Future Directions in the Regeneration of Multiple Tissues. Stem Cells International. 2020; 2020: 1-26.

[21] Kim M, Choi YS, Yang SH, Hong H, Cho S, Cha SM, et al. Muscle regeneration by adipose tissue-derived adult stem cells attached to injectable PLGA spheres. Biochemical and Biophysical Research Communications. 2006; 348: 386-392.

[22] Yoshida Y, Matsubara H, Fang X, Hayashi K, Nomura I, Ugaji S, et al. Adipose-derived stem cell sheets accelerate bone healing in rat femoral defects. PLoS ONE. 2019; 14: e0214488.

[23] Priyadarshini P, Samuel S, Kurkalli BG, Kumar C, Kumar BM, Shetty $\mathrm{N}$, et al. In vitro Comparison of Adipogenic Differentiation in Human Adipose-Derived Stem Cells Cultured with Collagen Gel and Platelet-Rich Fibrin. Indian Journal of Plastic Surgery. 2021; 54: 278-283.

[24] Yin F, Cai J, Zen W, Wei Y, Zhou W, Yuan F, et al. Cartilage Regeneration of Adipose-Derived Stem Cells in the TGF- $\beta 1$ Immobilized PLGA-Gelatin Scaffold. Stem Cell Reviews and Reports. 2015; 11: 453-459.

[25] Yamamoto D, Tada K, Suganuma S, Hayashi K, Nakajima T, Nakada M, et al. Differentiated adipose-derived stem cells promote peripheral nerve regeneration. Muscle \& Nerve. 2020; 62: $119-127$.

[26] Guo J, Guo S, Wang Y, Yu Y. Promoting potential of adipose derived stem cells on peripheral nerve regeneration. Molecular Medicine Reports. 2017; 16: 7297-7304.

[27] Rodriguez A-, Elabd C, Amri E, Ailhaud G, Dani C. The human adipose tissue is a source of multipotent stem cells. Biochimie. 2005; 87: 125-128.

[28] Mehrabani D, Mehrabani G, Zare S, Manafi A. Adipose-Derived Stem Cells (ADSC) and Aesthetic Surgery: a Mini Review. World Journal of Plastic Surgery. 2013; 2: 65-70.

[29] Nilforoushzadeh M A, Heidari-Kharaji M, Alavi S, Nouri M, Nikkhah N, Jahangiri F, et al. Transplantation of autologous fat, stromal vascular fraction (SVF) cell, and platelet-rich plasma (PRP) for cell therapy of atrophic acne scars: Clinical evaluation and biometric assessment. Journal of Cosmetic Dermatology. 2021. (in press)

[30] Sirowanto I, Josh F, Sulmiati, Ahmadwirawan, Zainuddin AA, Faruk M. The effect of Platelet-Rich Plasma and Stromal Vascular Fraction combination on Epidermal Growth Factor serum level for anal trauma healing in the Wistar rat model. Annals of Medicine and Surgery. 2021; 70: 102773.

[31] Pak C S, Heo C Y, Shin J, Moon S Y, Cho S W, Kang H. J. Effects of a catechol-functionalized hyaluronic acid patch combined with human adipose-derived stem cells in diabetic wound healing. International Journal of Molecular Sciences. 2021; 22:
2632.

[32] Virzì F, Bianca P, Giammona A, Apuzzo T, Di Franco S, Mangiapane LR, et al. Combined platelet-rich plasma and lipofilling treatment provides great improvement in facial skin-induced lesion regeneration for scleroderma patients. Stem Cell Research \& Therapy. 2017; 8: 236.

[33] Zhao F, Zhou L, Liu J, Xu Z, Ping W, Li H, et al. Construction of a vascularized bladder with autologous adipose-derived stromal vascular fraction cells combined with bladder acellular matrix via tissue engineering. Journal of Tissue Engineering. 2019; 10: 204173141989125.

[34] Paganelli A, Tarentini E, Benassi L, Scelfo D, Pisciotta A, Rossi $\mathrm{E}$, et al. Use of confocal microscopy imaging for in vitro assessment of adipose-derived mesenchymal stromal cells seeding on acellular dermal matrices: 3D reconstruction based on collagen autofluorescence. Skin Research and Technology. 2021. (in press)

[35] Murata D, Arai K, Nakayama K. Scaffold-Free Bio-3D Printing Using Spheroids as "Bio-Inks" for Tissue (re-)Construction and Drug Response Tests. Advanced Healthcare Materials. 2020; 9: 1901831.

[36] Watanabe M, Ohyama A, Ishikawa H, Tanaka A. Threedimensional bone formation including vascular networks derived from dental pulp stem cells in vitro. Human Cell. 2019; 32: $114-124$

[37] Kalra A. Developing FE Human Models from Medical Images. Basic Finite Element Method as Applied to Injury Biomechanics. 2018; 124: 389-415.

[38] Guimarães CF, Gasperini L, Marques AP, Reis RL. The stiffness of living tissues and its implications for tissue engineering. Nature Reviews Materials. 2020; 5: 351-370.

[39] Weiner S, Traub W, Wagner HD. Lamellar Bone: StructureFunction Relations. Journal of Structural Biology. 1999; 126: 241-255.

[40] Wang W, Yeung KWK. Bone grafts and biomaterials substitutes for bone defect repair: a review. Bioactive Materials. 2017; 2: 224-247.

[41] Nakamura A, Murata D, Fujimoto R, Tamaki S, Nagata S, Ikeya $\mathrm{M}$, et al. Bio-3D printing iPSC-derived human chondrocytes for articular cartilage regeneration. Biofabrication. 2021; 13: 044103.

[42] Murata D, Tokunaga S, Tamura T, Kawaguchi H, Miyoshi N, Fujiki M, et al. A preliminary study of osteochondral regeneration using a scaffold-free three-dimensional construct of porcine adipose tissue-derived mesenchymal stem cells. Journal of Orthopaedic Surgery and Research. 2015; 10: 35.

[43] Murata D, Akieda S, Misumi K, Nakayama K. Osteochondral Regeneration with a Scaffold-Free Three-Dimensional Construct of Adipose Tissue-Derived Mesenchymal Stromal Cells in Pigs. Tissue Engineering and Regenerative Medicine. 2018; 15: 101-113.

[44] Yamashita A, Morioka M, Yahara Y, Okada M, Kobayashi T, Kuriyama S, et al. Generation of scaffoldless hyaline cartilaginous tissue from human iPSCs. Stem Cell Reports. 2015; 4: 404-418.

[45] Anada T, Pan C, Stahl AM, Mori S, Fukuda J, Suzuki O, et al. Vascularized Bone-Mimetic Hydrogel Constructs by 3D Bioprinting to Promote Osteogenesis and Angiogenesis. International Journal of Molecular Sciences. 2019; 20: 1096.

[46] Byambaa B, Annabi N, Yue K, Trujillo-de Santiago G, Alvarez MM, Jia W, et al. Bioprinted Osteogenic and Vasculogenic Patterns for Engineering 3D Bone Tissue. Advanced Healthcare Materials. 2017; 6.

[47] Shuai C, Yang W, Peng S, Gao C, Guo W, Lao Y, et al. Physical stimulations and their osteogenesis-inducing mechanisms. International Journal of Bioprinting. 2018; 4: 138. 